\title{
The Coupling Model for Function and Delay Faults
}

\author{
JOONHWAN YI \\ Telecommunication Research Center, Samsung Electronics Corporation, Suwon, Kyunggi-Do, Republic of Korea \\ joonhwan.yi@samsung.com \\ JOHN P. HAYES \\ Department of Electrical Engineering and Computer Science, University of Michigan, 1301 Beal Avenue, \\ Ann Arbor, MI 48109, USA \\ jhayes@eecs.umich.edu
}

Received June 11, 2003; Revised July 6, 2005

Editor: V. Agrawal

\begin{abstract}
We propose a high-level fault model, the coupling fault (CF) model, that aims to cover both functional and timing faults in an integrated way. The basic properties of CFs and the corresponding tests are analyzed, focusing on their relationship with other fault models and their test requirements. A test generation program COTEGE for CFs is presented. Experiments with COTEGE are described which show that (reduced) coupling test sets can efficiently cover standard stuck-at-0/1 faults in a variety of different realizations. The corresponding coupling delay tests detect all robust path delay faults in any realization of a logic function.
\end{abstract}

Keywords: delay faults, fault modeling, functional faults, test generation

\section{Introduction}

Gate-level faults, such as the standard stuck-at line (SSL) and path delay fault models, represent faulty behavior associated with logic gates and their interconnections. Consequently, they are not well suited to designs that contain higher-level modules whose implementation details are unavailable. In such cases, we need to check the faulty behavior of a module with respect to the module's overall specifications, and high-level fault models can play an important role. In

This research was sponsored in part by the U.S. National Science Foundation under Grants No. CCR-9872066 and CCR-0073406. A shorter version of this paper was published in the Proceedings of European Test Workshop, 2001. The single coupling fault (CF) and coupling delay fault (CDF) models of the earlier paper are extended here to the general CF and CDF models, and more analysis and discussion are presented. addition, high-level testing can be used to correct testability problems in early design stages [19], or to test multiple implementations of a high-level design [14]. A module's specification is typically in the form of a Boolean expression, a truth table, a binary decision diagram, or a behavioral Verilog code description.

Several higher-level fault models have been proposed for realization-independent functional testing of arbitrary combinational modules, including the cell and pin fault models. A cell fault [20] implicitly models all defects that alter a module's specification and so provides a high degree of realization independence. This model can only be applied to very small modules, however, because it requires an exhaustive test set comprising all possible input vectors (patterns). For example, in the case of the $n$-bit $A D D E R$ module shown in Fig. 1(a), $2^{2 n+1}$ tests are required to detect all cell faults. The pin fault model considers stuck-at-0/1 faults occurring at 


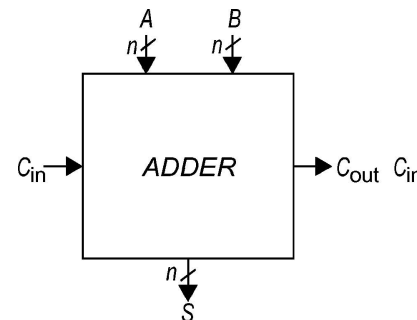

(a)

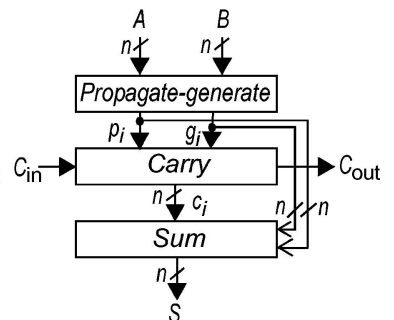

(b)
Fig. 1. (a) An $n$-bit adder module. (b) High-level structure of a carry-lookahead adder [14].

the module boundary, and has weak correlation with the circuit's physical faults. For $A D D E R$, just two tests, the all- 0 and all- 1 vectors, suffice to detect the $6 n+4$ possible pin faults. The SSL model lies somewhere between cell and pin faults, but is realization-dependent. To detect all SSL faults in a two-level implementation (not a practical design) of the $n$-input adder, $2^{2 n+1}$ tests, that is, exhaustive testing, is required. However, $2 n+2$ tests are sufficient to detect SSL faults in various practical adder implementations [2].

Another interesting approach to high-level testing is based on the concept of a universal test set [1, 4]. It exploits the unateness (monotonicity) property of a module's variables, and is composed of functionallydefined minimal true and maximal false tests. A universal test set can detect both single and multiple stuck-line (MSL) faults in realizations with minimal restrictions on their structure [14]. The size of the universal test set is small for functions that are fully or partially unate, but it becomes exhaustive for binate functions. For example, the universal test set is exhaustive for the ADDER module of Fig. 1(a) because the $S$ (sum) outputs are binate in all $2 n+1$ input variables. If a circuit is decomposed into suitable submodules and universal test sets are applied to the submodules, as in the RIBTEC technique [14], the resulting functional tests achieve a high degree of realization independence with $O\left(n^{k}\right)$ tests for some small $k$. The modular $n$-input carry look-ahead adder shown in Fig. 1(b), for instance, requires far fewer than $2^{2 n+1}$ tests-it requires 44 tests when $n=16$.

Few high-level delay fault models have been proposed so far. Pomeranz and Reddy [18] introduced the gross and function-robust delay fault models. As we show later in Section 4, the gross delay model covers all path delay faults. However, it requires all adjacent vector pairs as tests, which results in huge test sets $\left(n 2^{n}\right.$ tests for an $n$-input module). The function-robust delay fault model covers all robust path delay faults. Using the powerful functional fault coverage of a universal test set, Sparmann et al. [24] proposed the universal delay test set which, however, is only applicable to unate functions. Psarakis et al. [21] studied delay testing using what they call the realistic sequential cell fault model (RS-CFM). Their approach targets nonspecific "sequential" faults such as stock-open faults in special classes of circuits (hazard-free iterative logic arrays). The RS-CFM model is not suitable for path delay fault testing because it is defined for an iterative logic array in which at most a single cell (or module) is faulty.

Digital systems are susceptible to both functional and timing faults due to increasing operating speeds and decreasing process feature sizes. This paper proposes a fault model, the coupling fault (CF) model, that covers both functional and timing faults in an integrated way, and is applicable to fairly large modules without imposing significant design constraints. A CF models (static) functional faults, but is motivated by (dynamic) transition effects, and so is easily extendible to a delay fault model. The corresponding coupling test sets share some properties with universal test sets, but are not necessarily exhaustive for binate functions. They also achieve high SSL fault coverage for a wide range of implementations. A pair of adjacent coupling tests (CTs) constitutes a coupling delay test, so the combinational $\mathrm{CF}$ model naturally extends to the sequential $\mathrm{CF}$ model. We show that coupling delay tests, which happen to correspond to the single input change (SIC) tests used in [21] and elsewhere, achieve high coverage of path delay faults. We also show that the coupling delay test set (CDTS) for a function $z$ is a subset of other high-level delay test sets for $z$, e.g. the gross and function-robust delay test sets.

The size of a high-level test set is usually much larger than that of a realization-dependent one to assure good fault coverage for many realizations. Often this large size limits the application of high-level test sets to a small portion of a circuit. The interface region around the intellectual property (IP) circuits in a system-ona-chip (SOC) is a good candidate for high-level delay testing because its implementation details are not known to either the SOC or the IP designers. For example, consider a high-level combinational module $C U T$ at the interface of two $I P$ circuits $I P_{1}$ and $I P_{2}$ in an SOC $C$, as shown in Fig. 2. $C U T$ is composed of two modules, say $M_{1}$ and $M_{2}$, which are included in $I P_{1}$ 


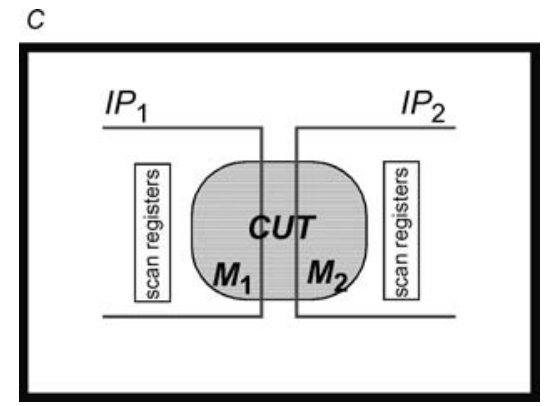

Fig. 2. A combinational module $C U T$ at the interface of two $I P$ circuits $I P_{1}$ and $I P_{2}$ in an SOC $C$.

and $I P_{2}$, respectively. Note that none of the designers of $I P_{1}$ and $I P_{2}$ knows the gate-level implementations of CUT fully because $M_{1}\left(M_{2}\right)$ is a high-level module to the designer of $I P_{2}\left(I P_{1}\right)$. It is thus very hard to detect faults in CUT using gate-level fault models. Designfor-testability (DFT) techniques [7, 13] can be used if the delay penalty due to DFT circuits is tolerable. Otherwise, high-level testing approaches appear very attractive if the scan design at IP circuit boundaries and high-level descriptions of $M_{1}$ and $M_{2}$ are known, which constitutes a very limited disclosure in the case of IPs.

Section 2 of this paper formalizes coupling faults and tests. Functional testing is examined in Section 3, while Section 4 considers delay testing. A coupling-test generation program COTEGE is presented in Section 5. Finally in Section 6, some experimental results on the fault coverage of coupling tests are presented.

\section{Coupling Faults and Tests}

Intuitively, a CF alters output values in response to changes occurring on one or more inputs of a logic function. The simplest case is a single $C F$ which is

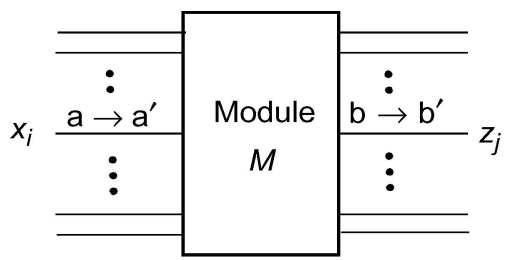

(a) defined in terms of a single input/output signal pair. In contrast, a general $C F$ is defined in terms of multiple inputs and a single output signal. Mainly single CFs are considered in this paper, and "CF" means a single $\mathrm{CF}$ unless otherwise noted.

Consider the module $M$ in Fig. 3 which realises $m$ Boolean functions $Z=\left\{z_{1}, z_{2}, \ldots, z_{m}\right\}$ with $n$ input variables $X=\left\{x_{1}, x_{2}, \ldots, x_{n}\right\}$. The normal "coupling relationship" between any $x_{i}$ in $X$ and any $z_{j}$ in $Z$ can make $z_{j}$ change from $b$ to its complement $b^{\prime}$, denoted $b \rightarrow b^{\prime}$, when $x_{i}$ undergoes the change $a \rightarrow a^{\prime}$ in some input vector $v$ applied to $M$; see Fig. 3(a). Any change to this relationship is modeled by a CF denoted by $x_{i} \mid z_{j}$. That is, when the transition $a \rightarrow a^{\prime}$ occurs in input $x_{i}$, the fault $x_{i} \mid z_{j}$ in $M$ either makes $z_{j}$ stable at $b$ or $b^{\prime}$, or else produces the inverted transition $b^{\prime} \rightarrow b$; see Fig. 3(b). Each of these possible faulty behaviors is revealed by some test vector that can propagate a signal transition from $x_{i}$ to $z_{j}$ when $M$ is fault-free. All such vectors are represented by the Boolean difference of $z_{j}$ with respect to $x_{i}$, which is defined as follows.

$$
\begin{aligned}
\frac{d z_{j}}{d x_{i}}= & z_{j}\left(x_{1}, \ldots, x_{i}=0, \ldots, x_{n}\right) \\
& \oplus z_{j}\left(x_{1}, \ldots, x_{j}=1, \ldots, x_{n}\right)
\end{aligned}
$$

Any vector $v$ making $\frac{d z_{j}}{d x_{i}}=1$ can be affected by a CF of the form $x_{i} \mid z_{j}$, leading to the following definition.

Definition 1 (Coupling Fault). A coupling fault $x_{i} \mid z_{j}$ from $x_{i}$ to $z_{j}$ in module $M$ changes the value of $z_{j}$ for at least one input vector satisfying $\frac{d z_{j}}{d x_{i}}=1$

Observe that this definition is realizationindependent and combinational (static); detection of a $\mathrm{CF}$ requires a single test vector not a vector-pair.

We refer to the set of all test vectors for a CF set $F$ as its coupling test set (CTS). We also follow the common practice of using a Boolean function $\operatorname{CTS}_{F}(X)$ to

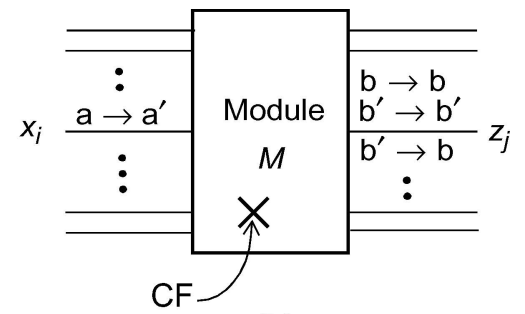

(b)

Fig. 3. (a) A fault-free combinational module $M$, and (b) $M$ with a coupling fault CF present. 
represent test sets, where $C T S_{F}(v)=1$ if and only if $v$ is a test for some fault in $F$. Each member of the CTS thus corresponds to a minterm of $C T S_{F}(X)$. To detect $x_{i} \mid z_{j}$, we need to apply all input vectors satisfying $\frac{d z_{j}}{d x_{i}}=1$. Therefore the set of all CTs for $x_{i} \mid z_{j}$ is given by

$$
\operatorname{CTS}_{x_{i} \mid z_{j}}(X)=\frac{d z_{j}}{d x_{i}}
$$

Each function $z_{j}(X)$ in $Z$ has $n$ CFs $x_{1}\left|z_{j}, x_{2}\right| z_{j}, \ldots, x_{n} \mid z_{j}$, so the CTS for all such faults $F$ in $Z$ is

$$
C T S_{F}(X)=\sum_{i} \sum_{j} \frac{d z_{j}}{d x_{i}}
$$

The $n$-input identity function $I_{n}$ is composed of two minterms separated by the maximum Hamming distance $n$. For example, $I_{4}=a b c d+a^{\prime} b^{\prime} c^{\prime} d^{\prime}$ and $C T S_{a \mid I_{4}}(a, b, c, d)$ can be represented by

$$
\frac{d I_{4}}{d a}=b c d+b^{\prime} c^{\prime} d^{\prime}
$$

Hence the corresponding CTS is $\{1111,0111,1000$, $0000\}$. The complete CTS for $I_{4}$ consists of the 10 vectors indicated by shading in Fig. 4.

The coupling test set $C T S_{x_{i} \mid z_{j}}(X)$ is independent of $x_{i}$ since $\frac{d z_{j}}{d x_{i}}$ is independent of $x_{i}$. This implies that if $v=x_{1} \ldots x_{i-1} 0 x_{i+1} \ldots x_{n}$ is a CT, so is the adjacent $C T v^{*}=x_{1} \ldots x_{i-1} 1 x_{i+1} \ldots x_{n}$ obtained by complementing $x_{i}$. Note that $v=x_{i}^{\prime} \cdot s$ and $v^{*}=x_{i} \cdot s$, where $s$ satisfies $\frac{d z_{j}}{d x_{i}}=1$. Thus, $z_{j}\left(v^{*}\right)=z_{j}^{\prime}(v)$ from the definition of the Boolean difference. For example, abcd is an adjacent CT to the CT $a^{\prime} b c d$ for input $a$ in $I_{4}$. Conversely, if two input vectors $v$ and $v^{*}$ are adjacent and yield different outputs, then $v$ and $v^{*}$ are adjacent

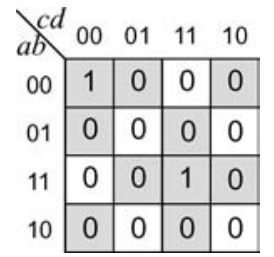

Fig. 4. The fourinput identity function $I_{4}=$ abcd + $a^{\prime} b^{\prime} c^{\prime} d^{\prime}$ with coupling tests shaded.
CTs. Clearly, all CTs lie on the boundary between the 0 's and 1 's in the $K$-map of the target function $z_{j}$; see Fig. 4. It also follows that the CTs completely define $z_{j}$.

A pair $\left\langle v, v^{*}\right\rangle$ of adjacent CTs for input $x_{i}$ and output $z_{j}$ can serve to detect a delay fault that causes excessive delay in propagating a signal transition on $x_{i}$ to $z_{j}$. The consecutive application of $v$ and $v^{*}$ activates a signal transition on $x_{i}$ which propagates through the module to $z_{j}$. As in conventional delay testing, we can check for an associated path delay fault by monitoring $z_{j}$ after applying $\left\langle v, v^{*}\right\rangle$.

Definition 2 (Coupling Delay Fault). A module $M$ contains a coupling delay fault $f=x_{i}-z_{j}$ from $x_{i}$ to $z_{j}$ if a signal transition on $x_{i}$ fails to propagate to $z_{j}$ within a specified time. The corresponding coupling delay test set (CDTS) is the set of all adjacent CT pairs $\left\langle v, v^{*}\right\rangle$ of $x_{i} \mid z_{j}$.

Suppose that the probability of a vector $v$ of $z$ being a minterm (or maxterm) is $2^{-1}$. Vector $v$ has $n$ adjacent vectors. If $v$ is not a CT, all $n$ adjacent vectors must produce the same output value $z(v)$. The probability that $v$ is not a CT is then $\left(2^{-1}\right)^{n}=2^{-n}$, that is, the probability that a vector of $z$ is a CT is $1-2^{-n}$ Since there are $2^{n}$ possible vectors, we conclude that the average size of $C T S_{z}$ is $2^{n} \cdot\left(1-2^{-n}\right)=2^{n}-1$.

Although the average size of a CTS is large, many useful functions require small CTSs. The elementary $n$-input (gate) functions, i.e. AND, NAND, OR, and NOR, require $n+1 \mathrm{CTs}$ which are identical to the minimal SSL fault test sets. This implies that test generation for CFs reduces to the standard ATPG problem when applied to gate-level circuits. Also, as shown in Section 6, many arithmetic functions (adders, ALUs and comparators) have CTSs of reasonable size.

Table 1 shows various coupling test sets for some elementary (gate) functions, including the identity function. In the XOR case, the CTS is exhaustive; a twolevel XOR circuit (usually impractical) requires all input vectors to detect all SSL faults. However, a practical $n$-input XOR tree circuit requires only four tests for any $n$ [11]. In the case of the identity function $I_{n}$, the CTS size increases linearly with $n$ and is identical to the SSL fault test set for a two-level sum-of-products (SOP) implementation of $I_{n}$. Observe that the universal test set for $I_{n}$ is exhaustive because $I_{n}$ is fully binate. Using the Synopsys Design Compiler, we synthesized two different implementations of the 20-input identity function $I_{20}$ as shown in Fig. 5; one is optimized for low area and the other for high speed. The conventional ATPG 
Table 1. Coupling test sets and their sizes for some basic Boolean functions.

\begin{tabular}{|c|c|c|c|c|c|}
\hline & & & \multicolumn{3}{|c|}{ Test set size } \\
\hline \multicolumn{2}{|c|}{$\begin{array}{c}\text { Function } \\
z\left(x_{1}, \ldots, x_{n}\right)\end{array}$} & $C T S_{z}$ & $\begin{array}{l}\text { Coupling } \\
\text { test set }\end{array}$ & $\begin{array}{l}\text { Coupling } \\
\text { delay test set }\end{array}$ & $\begin{array}{l}\text { Minimal SSL } \\
\text { fault test set }\end{array}$ \\
\hline \multirow[t]{2}{*}{ AND } & $n=2$ & $x_{1}+x_{2}$ & 3 & 4 & 3 \\
\hline & $n$ & $\begin{array}{c}x_{2} x_{3} \ldots x_{n}+x_{1} x_{3} \ldots x_{n}+\cdots+x_{1} x_{2} \ldots x_{n-2} x_{n} \\
+x_{1} x_{2} \ldots x_{n-1}\end{array}$ & $n+1$ & $2 n$ & $n+1$ \\
\hline \multirow[t]{2}{*}{ OR } & $n=2$ & $x_{1}^{\prime}+x_{2}^{\prime}$ & 3 & 4 & 3 \\
\hline & $n$ & $\begin{array}{c}x_{2}^{\prime} x_{3}^{\prime} \ldots x_{n}^{\prime}+x_{1}^{\prime} x_{3}^{\prime} \ldots x_{n}^{\prime}+\ldots+x_{1}^{\prime} x_{2}^{\prime} \ldots x_{n-2}^{\prime} x_{n}^{\prime} \\
+x_{1}^{\prime} x_{2}^{\prime} \ldots x_{n}^{\prime}-1\end{array}$ & $n+1$ & $2 n$ & $n+1$ \\
\hline \multirow[t]{2}{*}{ XOR } & $n=2$ & 1 & 4 & 8 & 4 \\
\hline & $n$ & 1 & $2^{n}$ & $n 2^{n}$ & $4(\mathrm{XOR}$ tree $)$ \\
\hline \multirow[t]{2}{*}{ Identity } & $n=4$ & $\begin{array}{c}x_{2} x_{3} x_{4}+x_{2}^{\prime} x_{3}^{\prime} x_{4}^{\prime}+x_{1} x_{3} x_{4}+x_{1}^{\prime} x_{3}^{\prime} x_{4}^{\prime}+x_{1} x_{2} x_{4} \\
+x_{1}^{\prime} x_{2}^{\prime} x_{4}^{\prime}+x_{1} x_{2} x_{3}+x_{1}^{\prime} x_{2}^{\prime} x_{3}^{\prime}\end{array}$ & 10 & 16 & 10 (SOP) \\
\hline & $n$ & $x_{2} x_{3} \ldots x_{n}+x_{2}^{\prime} x_{3}^{\prime} \ldots x_{n}^{\prime}+\cdots+x_{1} x_{2} \ldots x_{n-1}+x_{1}^{\prime} x_{2}^{\prime} \ldots x_{n-1}^{\prime}$ & $2 n+2$ & $4 n$ & $2 n+2(\mathrm{SOP})$ \\
\hline
\end{tabular}

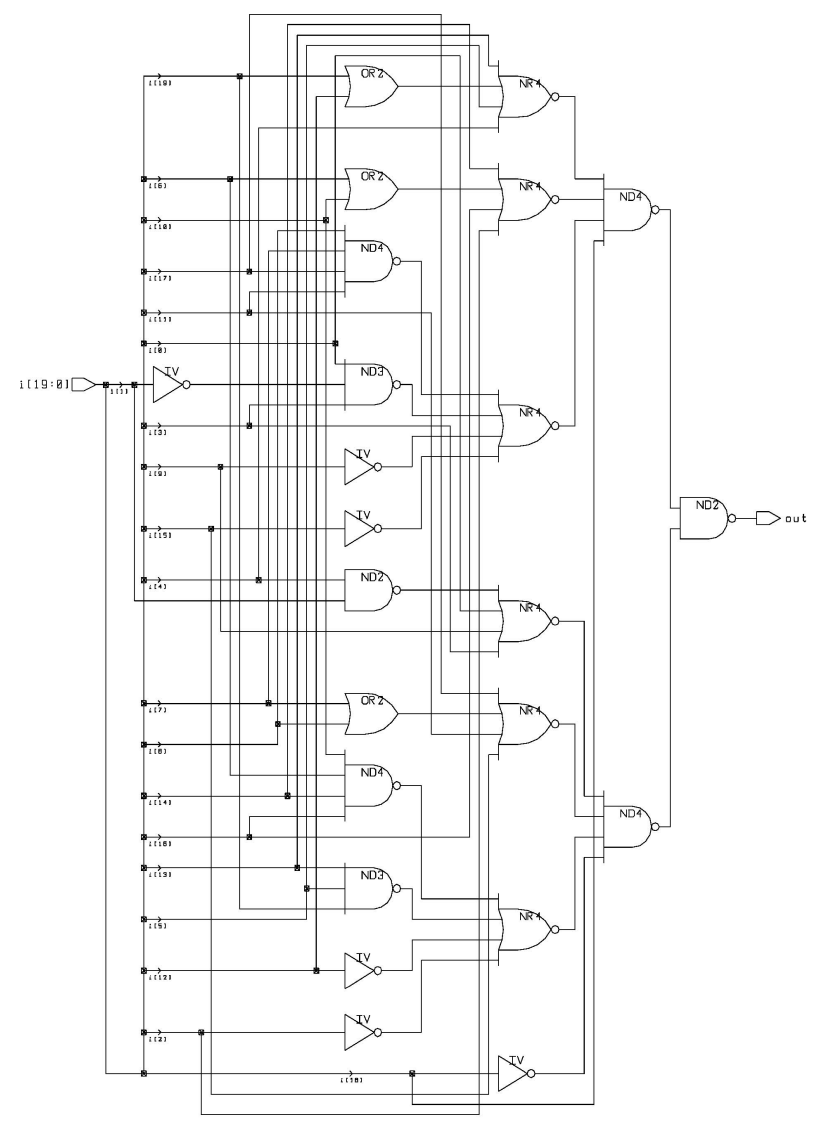

(a)

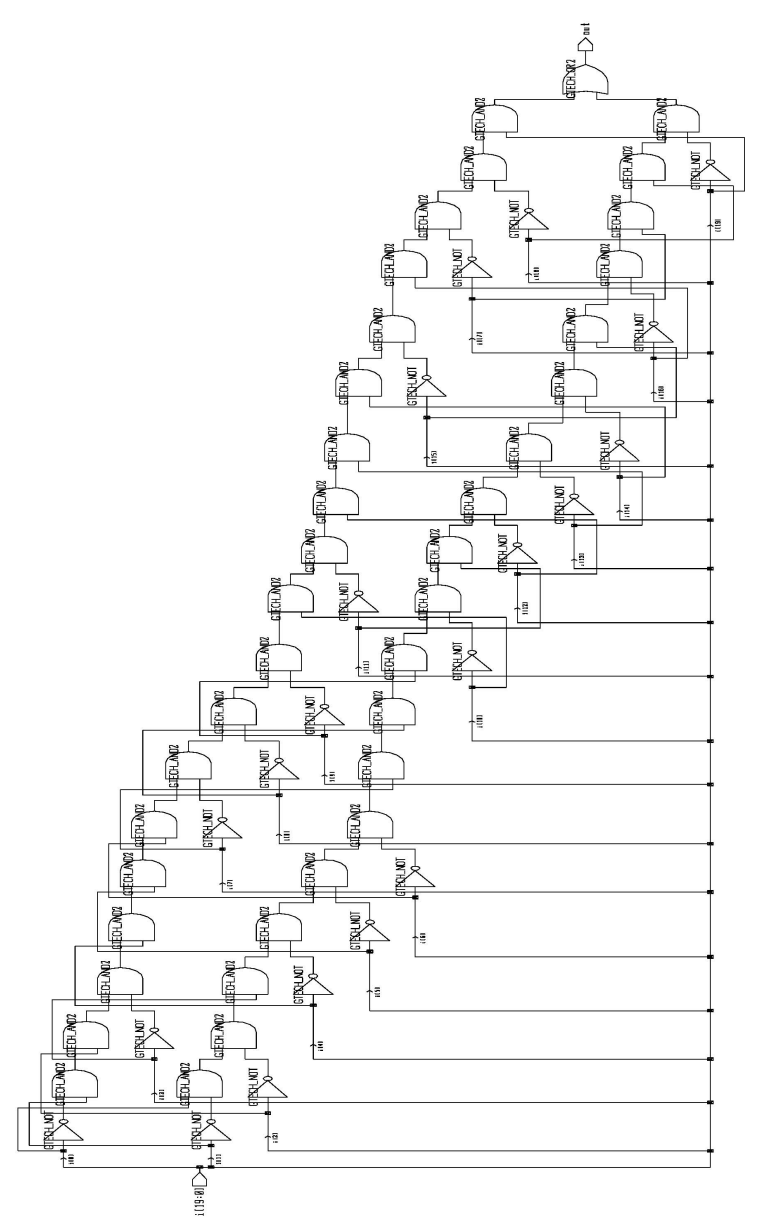

(b)

Fig. 5. Two gate-level realizations of the 20-input identity function $I_{20}$ synthesized for (a) high speed, and (b) low area. 
tool ATALANTA [16], generates 42 tests for both implementations; these tests happen to be identical to the CTs for $I_{20}$.

\subsection{General Coupling Faults and Tests}

Consider the module $M$ shown in Fig. 3 which realizes $m$ Boolean functions $Z=\left\{z_{1}, z_{2}, \ldots, z_{m}\right\}$ with $n$ input variables $X=\left\{x_{1}, x_{2}, \ldots, x_{n}\right\}$. A general CF $X_{i} \mid z_{j}$ blocks the coupling between a set of inputs $X_{i}=\left\{x_{i 1}, x_{i 2}, \ldots, x_{i k}\right\} \subseteq X$ and an output $z_{j}$ in $M$. Assume that $z_{j}$ changes $b \rightarrow b^{\prime}$ when a subset $X_{i}$ of $X$ changes from $A=l_{1} l_{2} \ldots l_{h} \ldots l_{k}$ to $B=l_{1}^{\prime} l_{2}^{\prime} \ldots l_{h}^{\prime} \ldots l_{k}^{\prime}$ where $l_{h}$ is either $x_{i h}$ or $x_{i h}^{\prime}$. If $M$ has CF $X_{i} \mid z_{j}$ then $z_{j}$ remains at $b$ or $b^{\prime}$, or else it changes $b^{\prime} \rightarrow b$ when $X_{i}$ changes $A \rightarrow B$. A general $\mathrm{CF}$ and its test set are similarly defined as follows.

Definition 3 (General Coupling Fault). A general coupling fault $X_{i} \mid z_{j}$ from a set of inputs $X_{i}$ to an output $z_{j}$ in module $M$ changes the value of $z_{j}$ for at least one input vector satisfying

$$
G=\frac{d}{d x_{i 1}}\left(\frac{d}{d x_{i 2}}\left(\cdots\left(\frac{d z_{j}}{d x_{i k}}\right)\right)\right)=1
$$

It follows that $C T S_{X_{i} \mid z_{j}}=G$ where $C T S_{X_{i} \mid z_{j}}$ is the CTS for $X_{i} \mid z_{j}$. A general CF $X_{i} \mid z_{j}$ is called a $k$-input $C F$ because $X_{i}$ is composed of $k$ inputs. For example, a single-input CF is a single CF. The CTS for all $k$-input CFs of $z_{j}$ contains all minterms $v$ and maxterms $v^{*}$ of $z_{j}$ where $v$ and $v^{*}$ are separated by Hamming distance $k$. That is, the general CTS for $z_{j}$ contains all minterms and maxterms separated by Hamming distances from 1 to $n$, which results in an exhaustive test set. Therefore, general CFs can model any physical defect that changes the function under test, like the cell fault model [20]. They provide strong realization independence but their application is restricted to small functions.

Consider a minterm-maxterm pair $t=\left\langle v, v^{*}\right\rangle$ where $v$ and $v^{*}$ are tests for $X_{i} \mid z_{j}$ and they are different in every input of $X_{i}$. Then $t$ can serve to detect a delay fault that causes excessive delay in signals that propagate from $X_{i}$ to $z_{j}$.

Definition 4 (General Coupling Delay Fault). A module $M$ contains a general coupling delay fault $X_{i}-z_{j}$ from $X_{i}$ to $z_{j}$ if any signal transitions on $X_{i}$ fail to propagate to $z_{j}$ within a specified time.

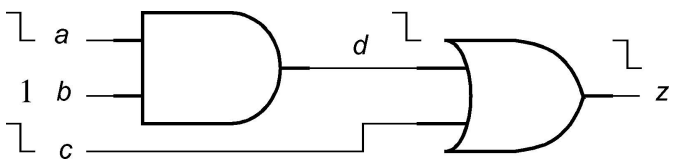

Fig. 6. The application of a test $t=\left\langle a b c, a^{\prime} b c^{\prime}\right\rangle$ to a gatelevel implementation of $z=a b+c$.

The corresponding general CDTS is the set of all minterm-maxterm pairs $\left\langle v, v^{*}\right\rangle$ where $v$ and $v^{*}$ are tests for $X_{i} \mid z_{j}$ and they are different in every input of $X_{i}$. A general coupling delay fault (CDF) $X_{i}-z_{j}$ is called a $k$-input $C D F$. The CDTS for all $k$-input CDFs of $z_{j}$ contains all minterm-maxterm pairs $\left\langle v, v^{*}\right\rangle$ separated by Hamming distance $k$. Thus, the general CDTS for $z_{j}$ is composed of all possible adjacent and non-adjacent minterm-maxterm pairs of $z_{j}$.

A multiple-input CDT $t$ propagates signal transitions through multiple input-output paths. Some single-input CDFs may be detected by $t$ and thus some singleinput CDTs can be replaced by $t$. For example, consider path delay faults in the implementation of function $z=a b+c$ appearing in Fig. 6. A two-input CDT $t=\left\langle a b c, a^{\prime} b c^{\prime}\right\rangle$ propagates falling signal transitions on $a$ and $c$ to $z$, and the latest signal transition on an input of OR gate will change $z$ from 1 to 0 . Therefore, $t$ detects slow-to-fall delay faults associated with both paths $a d z$ and $c z$. Since two single CDTs $t_{a}=\left\langle a b c^{\prime}, a^{\prime} b c^{\prime}\right\rangle$ and $t_{b}=\left\langle a^{\prime} b c, a^{\prime} b c^{\prime}\right\rangle$ also detect these two faults, $t_{a}$ and $t_{b}$ can be eliminated from the general CDTS of $z$. The relationship between singleand multiple-input CDTs is discussed further in [25].

\section{Properties of Coupling Tests}

In this section, coupling tests are related to test sets for SSL faults and high-level functional fault models. We derive a design constraint that ensures full coverage of SSL faults in a two-level circuit by the corresponding CTS.

A sum of products (SOP) cover $E$ of $z$ is a set of implicants $q_{1}, q_{2}, \ldots, q_{k}$ of $z$ such that $z=q_{1}+q_{2}+\cdots+q_{k}$. Each implicant $q_{i}$ corresponds to an AND gate in a twolevel AND-OR realization of $z$. An implicant $q_{i}$ of $E$ is relatively essential if the result of deleting $q_{i}$ from $E$ covers fewer minterms of $z$ than $E$. A cover is nonredundant if all implicants in the cover are relatively essential. Consider an implicant $q=l_{1} l_{2} \ldots l_{i} \ldots l_{k}$ in a non-redundant cover $E$ of $z$. Literal $l_{i}$ in $q$ is a prime literal [12] if the product term $r=l_{1} l_{2} \ldots l_{i-1} l_{i+1} \ldots l_{k}$ 
obtained by deleting $l_{i}$ from $q$ is not an implicant of $z$; clearly, all literals in a prime implicant are prime. An input vector $v$ is relatively essential [12] in $q$ if $q(v)=1$ but $q^{+}(v)=0$ for some other implicant $q^{+} \neq q$ in $E$. A relatively essential implicant contains at least one relatively essential vector. A cover corresponding to a non-redundant SOP realization of $z$ is composed of relatively essential prime implicants.

We now specify a design constraint to ensure that the CTS for $z$ detects all SSL faults in any nonredundant SOP realization $C$ of $z$. Let $E$ be the cover corresponding to $C$. Although we only consider SOP covers explicitly, the results hold for product-of-sums (POS) covers by the duality principle.

Lemma 1. A test set $T$ detects all SSL faults in a non-redundant SOP circuit $C$ if $T$ includes

(1) At least one relatively essential vector $v$ of every prime implicant $q$ in $E$, and

(2) At least one false vector $w$ adjacent to a minterm of $q$ for every literal $l_{i}$ in $q$.

Proof: Let AND gate $G$ in $C$ realize $q$ and let input $i$ of $G$ correspond to $l_{i}$. The proof is done by showing that $v$ detects the fault $i$ stuck-at- 0 and $w$ detects $i$ stuckat-1. First, consider $i$ stuck-at- 0 . Input $v$ activates this fault by setting all inputs of $G$ to 1 and propagates an error to $z$ by setting the output of every other AND gate $G^{*} \neq G$ to 0 . Now consider $i$ stuck-at-1. Since $w$ is adjacent to a minterm of $q$ at $l_{i}, w$ sets $i$ to 0 (fault activation) and all other inputs of $G$ to 1 . In addition, $w$ forces the output of $G^{*}$ to 0 because $w$ is a maxterm. That is, $w$ activates $i$ stuck-at- 1 and propagates a faulty value to $z$.

Every false vector adjacent to a minterm is a CT, that is, a CTS always satisfies condition (2) of Lemma 1. To meet condition (1), a CTS must contain at least one relatively essential vector from every prime implicant $q$ in $E$, which implies that $q$ must have at least one relatively essential vector (minterm) that is adjacent to a false vector (maxterm). This leads to the following sufficient condition for the CTS to detect all SSL faults in a two-level circuit

Theorem 1. Let $C$ be a non-redundant two-level circuit realizing an SOP cover $E$ of $z$. The coupling test set $C T S_{Z}$ detects all SSL faults in $C$ if every prime implicant in $E$ has at least one relatively essential vector that is adjacent to a false vector.

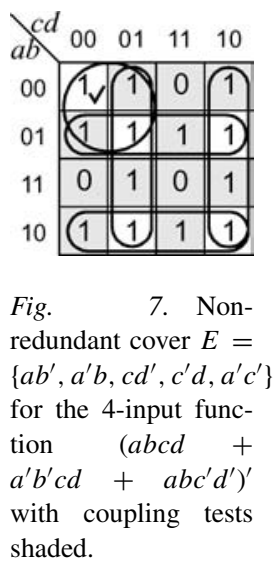

A two-level circuit that is testable for robust path delay faults [8] satisfies the design constraint specified in Theorem 1. Although it appears to be uncommon, some functions have non-redundant covers that fail to satisfy this constraint. For example, $I_{4}$ has some non-redundant POS covers that violate the constraint although all minimal SOP and POS covers satisfy it. Another example is shown in Fig. 7. Here the non-redundant cover $E=\left(a b^{\prime}, a^{\prime} b, c d^{\prime}, c^{\prime} d, a^{\prime} c^{\prime}\right)$ is not fully testable by the function's CTS. The checked vector $a^{\prime} b^{\prime} c^{\prime} d^{\prime}$ is an essential SSL test for the two-level circuit realizing $E$ but is not a CT. However, there is another minimal POS cover $\left(a^{\prime}+b^{\prime}+c^{\prime}+d^{\prime}, a+b+c^{\prime}+d^{\prime}, a^{\prime}+b^{\prime}+c+d\right)$ for this function that meets the foregoing constraint. We also found a 6-input function all of whose nonredundant covers violate this constraint:

$$
\begin{aligned}
z= & a b^{\prime} e f+a^{\prime} b e f+c d^{\prime} e f+c^{\prime} d e f+a c e f \\
& +c^{\prime} d^{\prime} e f^{\prime}+c d e f^{\prime}+c^{\prime} d^{\prime} e^{\prime} f+c d e^{\prime} f \\
& +a^{\prime} b^{\prime} c^{\prime} d e^{\prime} f^{\prime}+a^{\prime} b^{\prime} c d^{\prime} e^{\prime} f^{\prime}+a b c d^{\prime} e^{\prime} f^{\prime}
\end{aligned}
$$

The coupling test set $C T S_{Z}$ for $z$ has at least one relatively essential vector from 11 out of 12 prime implicants, which implies that most SSL faults are detected by $C T S_{Z}$. In fact, $C T S_{Z}$ detects 91 faults out of 92 possible (collapsed) faults in all eight possible SOP realizations of $z$.

Most practical circuits are not SOP realizations. A test-set preserving logic transformation [3] $L$ for a fault type $F$ transforms a circuit $N_{1}$ to $N_{2}$ denoted $L\left(N_{1}\right)=N_{2}$ such that a test set for $N_{1}$ detects all faults of type $F$ in $N_{2}$. Some important logic synthesis techniques such as algebraic factorization and resubstitution are test-set-preserving for SSL faults [22]. 


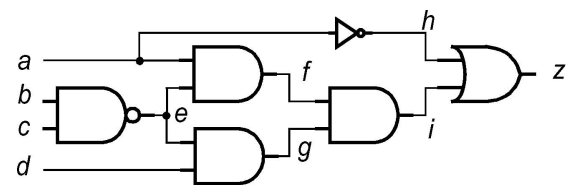

(a)

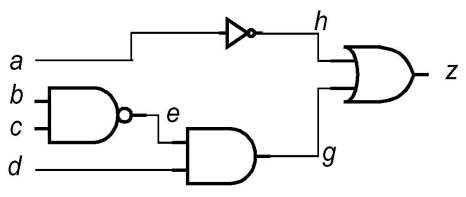

(b)

Fig. 8. (a) A non-BIP implementation $C_{0}$ of the function $z=a^{\prime}+b^{\prime} d+c^{\prime} d$, and (b) the BIP implementation $C_{0}^{*}$ of $z$ obtained by removing gates and lines from $C_{0}$.

Let $C$ be a two-level realization of a function $z$ that meets the design constraint given in Theorem 1 . Then, the coupling test set for $z$ also detects all SSL faults in a multiple-level circuit derived from $C$ by test-setpreserving synthesis techniques. As will be seen in our experimental results, the coupling test sets show high SSL fault coverage for a wide range of multilevel implementations.

CFs are also related to other existing fault models. Although pin faults are weak, as observed in Section 1, pin fault tests sometimes detect hard-to-detect (random-test resistant) faults. For example, the two all0 and all- 1 pin fault tests for an $n$-bit adder sensitize the long internal path from $C_{\text {in }}$ to $C_{\text {out }}$ used for carry propagation. In general, we can rewrite (2) as

$$
C T S_{x_{i} \mid z_{j}}(X)=x_{i} \frac{d z_{j}}{d x_{i}}+x_{i}^{\prime} \frac{d z_{j}}{d x_{i}}
$$

Since $x_{i} \frac{d z_{j}}{d x_{i}}$ represents all tests that detect $x_{i}$ stuck-at-0 via $z_{j}$ and $x_{i}^{\prime} \frac{d z_{j}}{d x_{i}}$ represents all tests that detect $x_{i}$ stuckat-1 via $z_{j}, C T S_{x_{i} \mid z_{j}}(X)$ must contain all tests for the SSL faults on $x_{i}$. Hence $C T S_{x_{i} \mid z_{j}}(X)$ includes all tests for pin faults.

Finally, we establish a relationship between coupling tests and the universal test set (UTS) $[1,4]$ for unate functions. The UTS covers all SSL faults in realizations that meet minor and practical constraints on inversion parity, such as the balanced inversion parity (BIP) constraint, and the slightly more restrictive unate-gate network constraint [14]. A BIP network is a gate-level circuit in which, whenever possible, all paths from any input variable to an output have the same inversion parity-the inversion parity of a path is $N$ modulo 2 , where $N$ is the total number of inversions on the path.

Consider a function $z$ whose positive and negative cofactors with respect to a variable $x$ are respectively denoted by $\left.z\right|_{x}$ and $\left.z\right|_{x^{\prime}}$ hence $z=\left.x \cdot z\right|_{x}+\left.x^{\prime} \cdot z\right|_{x^{\prime}}$. Function $z$ is positive unate in $x$ if $\left.\left.z\right|_{x} \supseteq z\right|_{x^{\prime}}$ and negative unate if $\left.\left.z\right|_{x} \subseteq z\right|_{x^{\prime}}$. Otherwise, $z$ is binate in $x$. Function $z$ is unate if all its variables are unate; otherwise, $z$ is binate. It is shown in [14] that any logic function $z$ can be realized such that all paths from a unate variable of $z$ have the same inversion parity, while at least one path from a binate variable must have a different inversion parity from other paths. BIP realizations cover a broad range of implementations and tend to be minimal or near-minimal in gate count because any inversion that violates the BIP condition can be easily removed without adding gates. The authors of [14] report that all synthesized (gate-level) circuits used in their experiments meet the BIP condition with no design changes. For example, Fig. 8(a) is a non-BIP implementation $C_{0}$ of the function $z=a^{\prime}+b^{\prime} d+c^{\prime} d$ because there is an even inversion path afiz from $a$ to $z$, and $z$ is negative unate in $a$. The BIP implementation $C_{0}^{*}$ of $z$ shown in Fig. 8(b) is obtained by removing the line between $a$ and $f$ two redundant gates from $C_{0}$.

An input vector $v_{i}$ is greater than or equal to another vector $v_{j}$, denoted $v_{i} \geq v_{j}$, if $v_{i}$ has 1 in every bit position where $v_{j}$ does. For example, $1110 \geq 0100$. The minimal true vectors $\left(v_{i}\right)$ of $z$ are input vectors such that $z\left(v_{j}\right)=1$ for all $v_{j} \geq v_{i}$, but $z\left(v_{j}\right)=0$ for all $v_{j}<v_{i}$. Maximal false vectors are defined similarly. Expanded vectors contain all input literals, both true and complemented, appearing in a function's minimal SOP expression [1]. For example, consider a function $z(a, b, c)=a b+a^{\prime} c^{\prime}$ that is binate in $a$, positive unate in $b$, and negative unate in $c$. The expanded vector of $a b c=111$ is $a a^{\prime} b c^{\prime}=1010$.

The UTS for a function $z$ is composed of all its maximal false and minimal true expanded vectors. It is shown in [4] that the UTS is minimal in the sense that no test set detects all SSL faults in a non-redundant two-level realization of a positive unate function with fewer tests than the UTS.

Theorem 2. The coupling test set CTS for a unate function $z$ contains the universal test set $U T S_{z}$ for $z$, that is, $C T S_{z} \supseteq U T S_{z}$. 
Proof: Without loss of generality, assume that $z$ is positive unate in all its variables. Then the UTS for $z$ is composed of all maximal false and minimal true vectors of $z$, and every minterm $m$ of $z$ must be expressible as a product containing at least one uncomplemented literal. Assume that $m$ is a minimal true vector that has no adjacent false vector. Then $m$ is surrounded by true vectors, so an adjacent true vector $m^{*}$ must be obtained by complementing an uncomplemented literal from $m$. Since $z$ is positive unate, $m>m^{*}$. This contradicts to the assumption that $m$ is a minimal true vector. Therefore, $m$ must have an adjacent false vector. Similarly, every maximal false vector is also adjacent to at least one true vector. All adjacent false and true vectors are CTs, so all maximal false and minimal true vectors are CTs.

This result implies that the CTS detects all SSL faults in BIP-type realizations of a unate function. Typically, only small portions of useful circuits are unate. In the case of binate functions, there is no obvious relation between the CTS and the UTS. While the UTS is always exhaustive for fully binate functions, the CTS can be smaller, as demonstrated by the identity function $I_{n}$.

Theorem 2 also implies that a CTS may contain some unnecessary or redundant tests for BIP-type realizations. From the definition of a UTS, any true (false) expanded vector that is greater (less) than some other true (false) expanded vector is an unnecessary test for BIP circuits, regardless of the function's unateness. Let a CT $v$ be true if $v$ is a true vector and false if $v$ is a false vector. The expanded form of a CT is called an expanded CT. Then for BIP circuits, a CTS can be reduced by removing all true expanded CTs greater than other true expanded CTs, and all false expanded CTs less than other false expanded CTs.

Definition 5 (Reduced Coupling Test Set). The reduced CTS for a function $z$ is the set of minimal true and maximal false expanded CTs of $z$.

As will be seen in Section 6, reduced CTSs are used in our experiments, and they show high SSL fault coverage for a wide range of practical implementations.

\section{Properties of Coupling Delay Tests}

Path-based models are widely accepted as realistic models of delay faults in gate-level logic circuits. The conventional path delay model [23] associates faults with single paths. On the other hand, some approaches $[9,12]$ associate delay faults with both single and multiple paths. In this section, we relate the coupling delay fault model to the conventional path [23] and so-called primitive [12] delay fault models.

A single path $p$ is an alternating sequence of lines and gates from a primary input to a primary output. Consider a gate $G$ lying on $p$. An input $i$ of $G$ is called an on-path input of $p$ if $i$ is on $p$; otherwise, $i$ is an offpath input. A multiple path $P$ is a set of single-paths that share the same primary output [12]. A (single) path delay fault $f_{p}=\uparrow p(\downarrow p)$ increases circuit delay beyond some acceptable threshold when a signal transition is propagated through $p$, and causes a rising (falling) transition at the primary output of $p$. Tests for path delay faults are divided into two main categories: robust and non-robust. The application of a robust test $t$ for $f_{p}$ will cause an incorrect output to be measured in the presence of $f_{p}$, independent of the delays in the rest of the circuit under test; $f_{p}$ is then called a robust (path) delay fault. All other tests are non-robust and the corresponding faults are non-robust delay faults. A path $p$ is static sensitized by a vector $v$ if $v$ sets all off-path inputs of $p$ to non-controlling values. If $t=\left\langle v_{1}, v_{2}\right\rangle$ is a test for $f_{p}, p$ is static sensitized by $v_{2}$ and a proper signal transition is initiated at the input of $p$.

For example, $t_{n}=\left\langle a^{\prime} b, a b\right\rangle$ is a robust test for fault $\downarrow a d g h$ in the circuit of Fig. 9(a). The signal edge with an arrow represents the signal transition propagated through the path under test. As can be seen in Fig. 9(a), there are two possible output waveforms. In either case, if fault $\downarrow a d g h$ is present, $t_{n}$ detects the fault because the sampling time will precede the edge with the arrow, and the sampling value is 1 rather than the expected value 0 . On the other hand, $t_{n}$ is also a non-robust test for a fault $\downarrow a d f h$ as shown in Fig. 9(b). If the output is sampled at time $t_{1}$ or $t_{3}$, the fault is detected. However, if the output is sampled at time $t_{2}$, the fault is not detected. The glitch at the output $h$ exists under the assumption that paths $a c f h$ and $a d g h$ are fault-free. If either of these two paths is faulty, no falling edge at $h$ can be propagated through the path under test $a d f h$ and thus $t_{n}$ is invalidated.

The on- and off-path signal conditions for robust and non-robust delay tests are summarized in Table 2, which is adapted from tables in [5] and [17]. According to these conditions, a robust test causes a signal transition whose initial and final values are different at on-path inputs and thus at the path output. That is, 
(a)

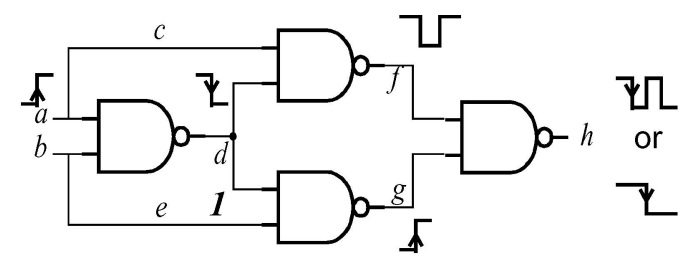

(b)

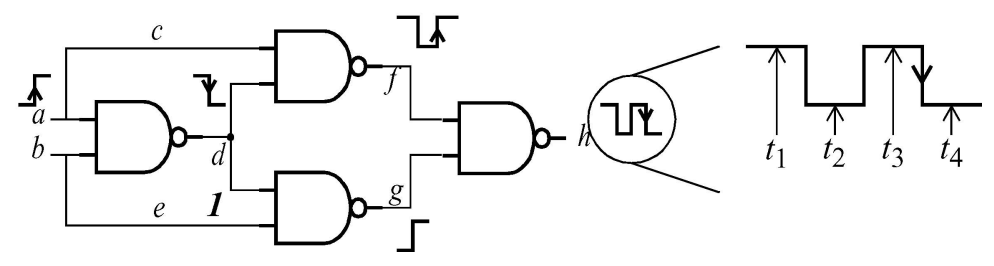

Fig. 9. A realization of the 2-input XOR function. Test $\left\langle a^{\prime} b, a b\right\rangle$ is (a) a robust test for $\downarrow a d g h$ and (b) a non-robust test for $\downarrow$ adfh.

two vectors in a robust test must yield different output values.

A non-robust test $t$ is said to be validatable nonrobust (VNR) if and only if there exists a set of tests $T$ including $t$ such that no element in $T$ is a robust test for the target fault $f_{p}$, and the correct response to $T$ implies that $f_{p}$ is not present in the circuit under test [12]. Robust and VNR tests guarantee detection of the target fault, while non-robust tests do not. Both robust and non-robust delay faults are called singly-testable (ST) faults in [9]. An ST-dependent fault is a path delay fault that does not cause excessive delay in the absence of a certain ST fault [9]. Thus, an ST-dependent fault does not need to be tested.

It is well-known that every detectable path delay fault has a single input change (SIC) test $t[9,23]$ in which the two input vectors of $t$ differ only in a single input variable.

Lemma 2. The set $T_{\text {SIC }}$ of all SIC tests is necessary and sufficient to detect all (single) path delay faults in any realization of a function $z$.

Table 2. Delay test classification in gate-level circuits. ( $c v$ and $n c v$ denote controlling and non-controlling values, respectively. $X$ denotes don't care values including glitches).

\begin{tabular}{lcc}
\hline & \multicolumn{2}{c}{ On-path Input } \\
\cline { 2 - 3 } Off-path input & $c v \rightarrow X \rightarrow n c v$ & $n c v \rightarrow X \rightarrow c v$ \\
\hline$X \rightarrow n c v$ & Robust & Non-robust \\
Stable $n c v$ & Robust & Robust \\
\hline
\end{tabular}

Proof: Sufficiency: By way of contradiction, assume that a path delay fault $f_{p}=\uparrow p$ or $\downarrow p$ only has a multiple-input-change test $t=\left\langle v_{1}, v_{2}\right\rangle$. This implies that at least one input $x_{k}$ in $t$ changes, where $x_{k}$ is not the primary input of $p$. Let $b_{1}$ and $b_{2}$ denote the values of $x_{k}$ in $v_{1}$ and $v_{2}$, respectively, where $b_{2}=b_{1}^{\prime}$. Then, $v_{2}$ must set all off-path inputs to non-controlling values. If $b_{2}$ makes every off-path input have a non-controlling value, the test $t^{*}=\left\langle v_{1}^{*}, v_{2}\right\rangle$ is also a test for $f_{p}$ where $v_{1}^{*}$ is obtained by replacing $b_{1}$ with $b_{2}$ in $v_{1}$. Similarly, we can make all other inputs except for the primary input of $p$ stable. That is, $f_{p}$ has an SIC test, which is contradictory to the initial assumption. So, every path delay fault has an SIC test and thus $T_{\text {SIC }}$ detects all path delay faults in any realization of $z$.

Necessity: This part of the proof follows from the fact that the sum-of-minterms realization of $z$ requires all SIC tests to detect all target faults.

Note that the necessary test set for gross delay faults is the set $T_{\mathrm{SIC}}$ of all SIC tests [19]. The CDTS $T_{\mathrm{CDF}}$ is the set of SIC tests of which the two vectors yield different outputs, that is, $T_{\mathrm{CDF}} \subseteq T_{\mathrm{SIC}}$. Also the test set $T_{\mathrm{FR}}$ for function-robust delay faults contains all SIC tests that yield different outputs [19], that is, $T_{\mathrm{CDF}} \subseteq T_{\mathrm{FR}}$.

Theorem 3. The CDTS for a function $z$ detects all robust path delay faults in any realization of $z$.

Proof: Consider a robust path delay fault $f_{r}=\uparrow p$ $(\downarrow p)$ in a realization of $z$. Then $f_{R}$ must have an SIC test $t_{R}=\left\langle v_{1}, v_{2}\right\rangle$ where $v_{1}$ and $v_{2}$ differ only in $p$ 's primary input. Note that $t_{R}$ propagates a signal transition 


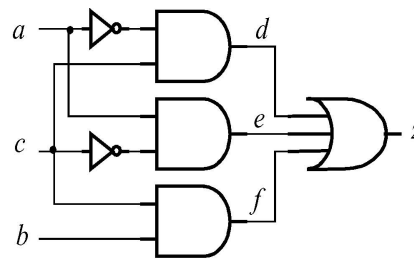

(a)

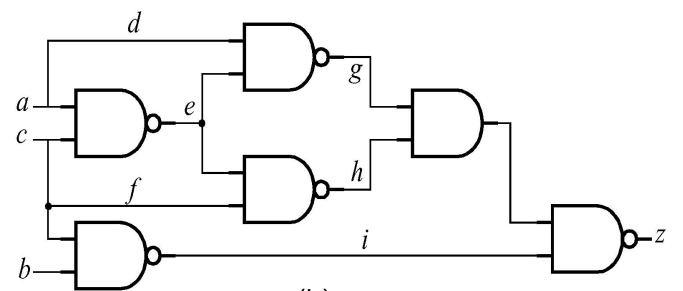

(b)

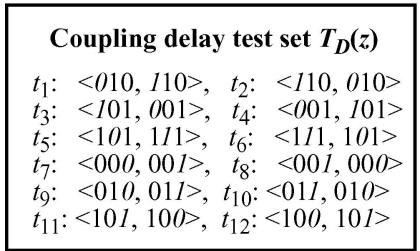

(c)

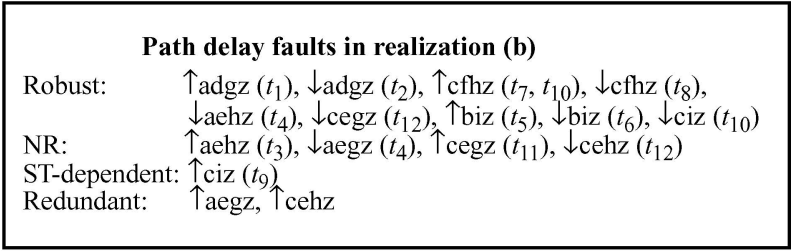

(d)

Fig. 10. (a) A 2-level BIP realization of $z=a c^{\prime}+a^{\prime} c+b c$, (b) a multi-level BIP realization of $z$, (c) coupling delay test set $T_{D}(\mathrm{z})$ for $z$, and (d) path delay faults and their tests (in parentheses) for realization (b).

through $p$ and causes a rising (falling) transition on the path output. Test $t_{R}$ is robust, so $v_{1}$ and $v_{2}$ must always yield different outputs. Since $t_{R}$ is an SIC test and $v_{1}$ and $v_{2}$ yield different output values, $t_{R}$ is a coupling delay test. Therefore the CDTS for $z$ detects $f_{R}$

It is worth noting that, in practical designs, the CDTS contains many non-robust tests. For example, Fig. 10 shows two realizations of the 3-input function $z=a c^{\prime}+a^{\prime} c+b c$ and the coupling delay test set $T_{D}(z)$. In the 2-level realization of Fig. 10(a), all 12 path delay faults are robust and are detected by $T_{D}(z)$. In the multi-level realization of Fig. 10(b), there are nine robust and four non-robust (NR) path delay faults with two redundant and one ST-dependent faults. All of these faults are detected by $T_{D}(z)$ as shown in Fig. 10(d). Although it is not necessary to test the ST-dependent fault $\uparrow c i z, T_{D}(z)$ has a test $t_{9}=$ $\langle 010,011\rangle$ that propagates a rising signal transition through path $\mathrm{ciz}$, to $z$, which may detect a fault in other realizations.

We now extend the result of Theorem 3 to transition faults and stuck-open faults. A (single) transition fault $f=\uparrow l(\downarrow l)$ on a line $l$ in a circuit $C$ causes excessive delays so that a rising (falling) signal transition on $l$ fails to propagate to any output $z_{j}$. Let $P_{l}$ be a partial path from $l$ to $z_{j}$. A transition fault test $t=\left\langle v_{1}, v_{2}\right\rangle$ for $f$ is composed of two input vectors $v_{1}$ and $v_{2}$ where $v_{2}$ static sensitizes $P_{l}$ and $t$ initiates a proper signal transition, that is a rising (falling) signal transition, on $l$. To make $t$ robust, all on- and off-path inputs of $P_{l}$ must meet the conditions given in Table 2. This implies that $v_{1}$ and $v_{2}$ must yield different outputs if $t$ is robust. Similarly to Lemma 2, we can prove that a robust transition fault has an SIC test. Because two vectors $v_{1}$ and $v_{2}$ in a robust test $t=\left\langle v_{1}, v_{2}\right\rangle$ yield different outputs, we can easily show that the CDTS of a function module $M$ detects all robust transition faults in any realization of $M$.

Theorem 4. The CDTS of a function $z$ detects all robust transition faults in any realization of $z$.

Consider a robust test $t$ for a path delay fault $f=\uparrow p$. Then, $t$ is a robust test for a transition fault associated with a line $l$ on $p$. For example, robust test $t_{1}=\langle 010,110\rangle$ for path delay fault $\uparrow a d g z$ in Fig. 10(b) is also a robust test for transition faults $\uparrow a, \uparrow d, \downarrow g$, and $\uparrow z$. The robust path delay tests $\left(t_{1}, t_{2}, t_{4}, t_{5}, t_{6}, t_{7}, t_{8}\right.$, $\left.t_{10}, t_{12}\right)$ in Fig. 10(d) robustly detect all transition faults except $\uparrow e$ in Fig. 10(b). The transition fault $\uparrow e$ is robustly detected by two CDTs $t_{3}$ and $t_{11}$ as can be seen in Fig. 11.

A stuck-open fault $f$ at a transistor $t r$ of a gate $G$ in a CMOS logic circuit can adversely affect the delay of $G$ in that a signal transition through $\operatorname{tr}$ can fail to propagate to an output $z_{j}$ through a partial path $P_{l}$ in the required time. Thus a test $t$ for $f$ is also composed of two input vectors. To make $t$ robust, all on- and off-path inputs of $P_{l}$ must meet the conditions given in 


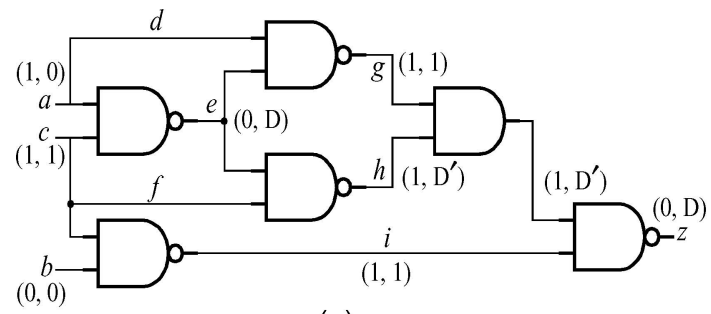

(a)

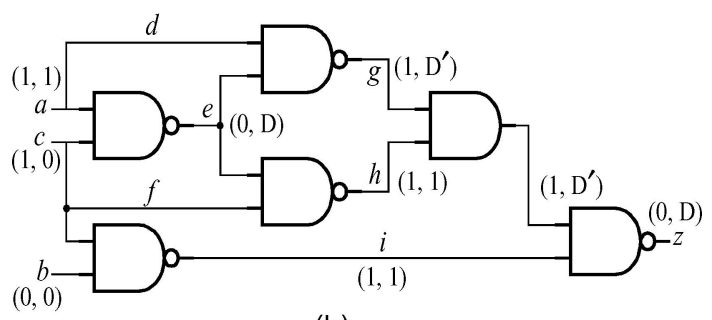

(b)

Fig. 11. Demonstration that transition fault $\uparrow e$ in Fig. 10(b) is robustly detected by two CDTs; (a) $t_{3}=\langle(101,001)\rangle$ and (b) $t_{11}=\langle(101,100)\rangle$.

Table 2. This implies that $v_{1}$ and $v_{2}$ must yield different outputs if $t$ is robust. As in the above discussion on the transition fault coverage of CDTSs, we can easily show that every stuck-open fault in a functional module $M$ has an SIC test, and thus the CDTS of $M$ detects all robust stuck-open faults in any realization of $M$.

Theorem 5. The CDTS of a function $z$ detects all robust stuck-open faults in any realization of $z$.

Next we derive a sufficient condition for a CDTS to detect primitive delay faults in two-level circuits. A multiple-path delay fault [12] $F_{P}=\uparrow P(\downarrow P)$ represents the condition in which every single path $p \in P$ has a fault $\uparrow p(\downarrow p)$. Multiple path $P$ is static sensitized by a vector $v$ if $v$ sets all side inputs of $P$ to noncontrolling values. $F_{P}$ is primitive if $P$ is static sensitizable, and there is no sub-path of $P$ that is similarly static sensitizable. Primitive delay faults include both robust and non-robust (single and multiple) path delay faults. If all primitive delay faults in a circuit $C$ have either a robust or a VNR test, these tests form a necessary and sufficient test set to guarantee that $C$ performs correctly at or below the test clock frequency [12].

We restrict our discussion to single-output circuits here. As defined in Section 3, a literal $l$ in an implicant $q$ of a function $z$ is prime if the result of deleting $l$ from $q$ is no longer an implicant of $z$. Consider a non-redundant two-level single-output circuit $C$ realizing a cover $E$ of $z$. Then, there are only two types of primitive delay faults according to [12]: $f_{1}=\downarrow P$, where multiple-path $P$ starts from a primary input associated with a prime literal $l_{i}$ in an implicant $q \in E$, and $f_{2}=\uparrow p$, where $p$ is a single path in $P$, i.e., $p \in P$. Let $v_{2}=l_{1} l_{2} \ldots l_{i} \ldots l_{n}$ be an input vector such that $q\left(v_{2}\right)=1$ and $v_{1}=l_{1} l_{2} \ldots l_{i}^{\prime} \ldots l_{n}$ be the result of replacing literal $l_{i}$ with $l_{i}^{\prime}$ from $v_{2}$. It is shown in [12] that the vector-pair $t_{m}=\left\langle v_{2}, v_{1}\right\rangle$ robustly detects $f_{1}$ when $E\left(v_{1}\right)=0$. It is also shown that $t_{s}=\left\langle v_{1}, v_{2}\right\rangle$ is a robust or a VNR test for $f_{2}$ when $v_{2}$ is a relatively essential vector of $q$ and $E\left(v_{1}\right)=0$. In other words, $t_{m}$ is composed of a true vector $v_{2}$ and the adjacent false vector $v_{1}$; thus $v_{1}$ and $v_{2}$ form a CT pair. If $C$ meets the conditions of Theorem 1, there exists at least one relatively essential vector $v_{2}$ in an implicant $q \in E$ that is adjacent to a false vector $v_{1}$, so that $\left\langle v_{1}, v_{2}\right\rangle$ detects $f_{2}$. Note that $v_{1}$ and $v_{2}$ are adjacent CTs.

Theorem 6. Consider a non-redundant two-level circuit $C$ realizing a cover $E$ of a single-output function z. The CDTS for $z$ contains a robust or a VNR test for every primitive delay fault in $C$ if and only if each implicant in $E$ has a relatively essential vector adjacent to a false vector.

The synthesis techniques algebraic factorization and resubstitution preserve primitive fault testability and primitive fault test sets [12]. Using this fact, we can extend Theorem 6 to multiple-level circuits. Hence, the coupling delay test set for $z$ also detects all primitive delay faults in a multi-level circuit derived from $C$ by using algebraic factorization and resubstitution.

\section{Coupling Test Generation}

COTEGE (COupling TEst GEnerator) is a test generation program for coupling faults that we developed for combinational modular circuits whose functional behavior is specified hierarchically as in RIBTEC [14]. As illustrated in Fig. 12, the combinational circuit of interest $C$ can be a sub-circuit (e.g. an adder) of a sequential circuit partitioned by registers, and each circuit $C$ is further partitioned into modules, e.g. a carry module of an adder. The CTS for each module is computed by COTEGE with respect to the module boundaries. Then 


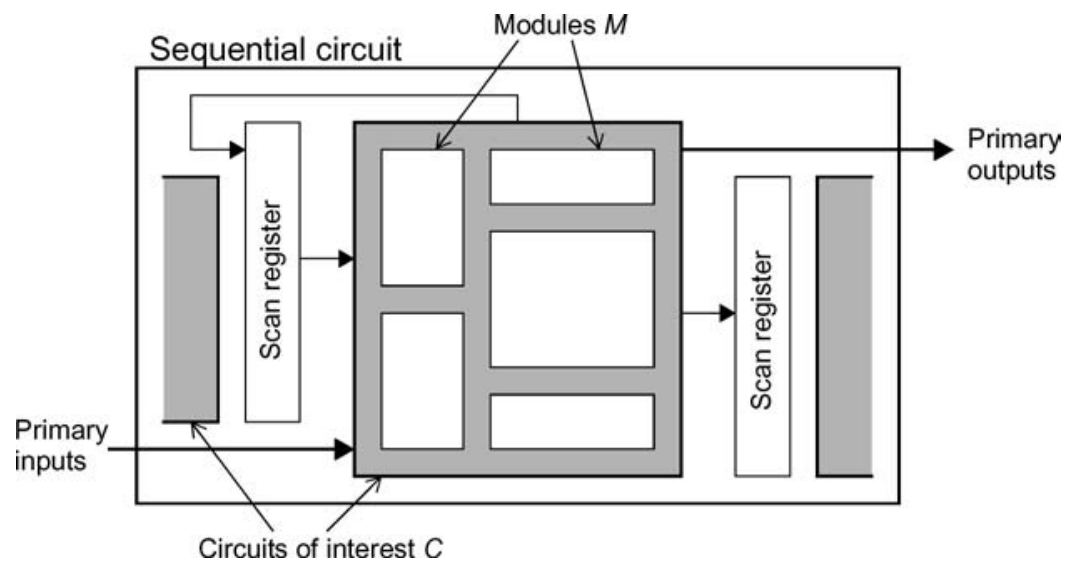

Fig. 12. Hierarchical specification of a circuit.

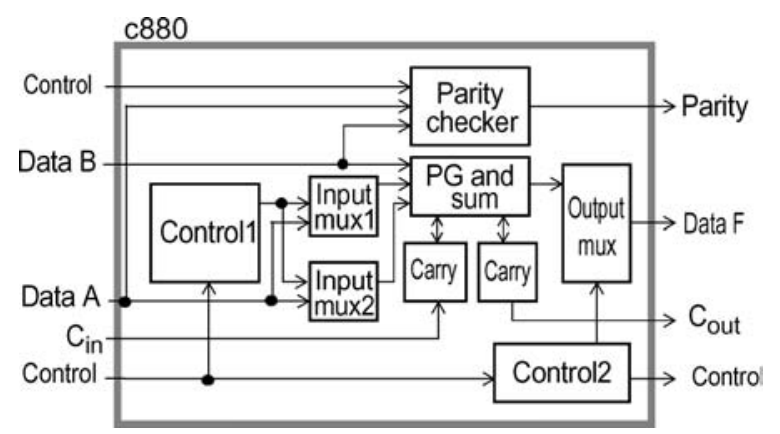

Fig. 13. Modular design of the c880 benchmark, an 8-bit ALU [14].

a test set $T$ for $C$ is generated with respect to the inputs of $C$ by using high-level techniques [14] based on PODEM [10]. Thus while $T$ is dependent on the modular interconnection structure of $C$, it is independent of each module's internal realization. Note that some CTs for an embedded module $m$ may not be applicable by $T$ because the inputs of $m$ depend on the functions of preceding modules. For example, Fig. 13 shows the modular structure of an ISCAS-85 benchmark circuit c880 used in our experiments. Circuit c880 is an 8-bit ALU, and is partitioned into various modules including carry, control, and parity-check modules.

COTEGE reads behavioral Verilog code defining a target circuit in sum-of-products form and generates a test output file which can be directly used by a conventional SSL fault simulator FSIM [16]. Fig. 14 gives a pseudocode description of COTEGE. The CTS $C T S_{k}$ for each type of module $M_{k}$ is generated by procedure $C T \operatorname{gen}()$ whose pseudocode is shown in Fig. 15. The reduced CTS $C T S_{k}^{R}$ is obtained from $C T S_{k}$ by removing all non-maximal false and non-minimal true CTs. Then for each module CT $t_{i j} \in C T S_{k}^{R}$, a high-level ATPG technique HL-PODEM [14] computes the test $p t$ defined at inputs of the circuit under test $C$. The fault simulation step removes any untested CFs that are detected by $p t$. After all CFs are detected, reverseorder fault simulation is performed for test compaction purposes.

The procedure HL-PODEM regards module input values of $t_{i j}$ and faulty responses $r_{i j}$ at the outputs of module $M_{k}$ as objectives in the standard PODEM algorithm, and computes an input vector $p t$ that applies $t_{i j}$ and propagates the faulty responses to a primary output. CT $t_{i j}$ is uncontrollable if no input vector of $C$ applies $t_{i j}$ to $M_{k}$. Since $M_{k}$ usually has multiple outputs and $t_{i j}$ can be a CT for the CF between another input-output pair, there can be several possible faulty responses $r_{i j}$ for $t_{i j}$. CT $t_{i j}$ is unobservable if the faulty value at the target module output $z_{j}$ cannot be propagated to a primary output of $C$ for at least one of all those faulty responses.

For every CT $t_{i j}$ that is uncontrollable and/or unobservable, HL-PODEM keeps replacing $t_{i j}$ with a set $T_{C}$ of covered CTs by $t_{i j}$ according to the universal test method of RIBTEC [14] until there exists no such $T_{C}$. Two vectors $t_{a}$ and $t_{b}$ are said to be comparable if either $t_{a} \geq t_{b}$ or $t_{a} \leq t_{b}$; otherwise, they are incomparable. Let $e_{a}$ denote the expanded vector of $t_{a}$. If $t_{i j}$ is a true vector, $T_{C}$ is the largest set $e_{k}$ of incomparable expanded true CTs that contains a CT $e_{k} \leq e_{h}$ for any true expanded CT $e_{h}>e_{i j}$. That is, every expanded CT $e_{h}$ greater than $e_{i j}$ has an expanded CT $e_{k} \leq e_{h}$. Hence, it 


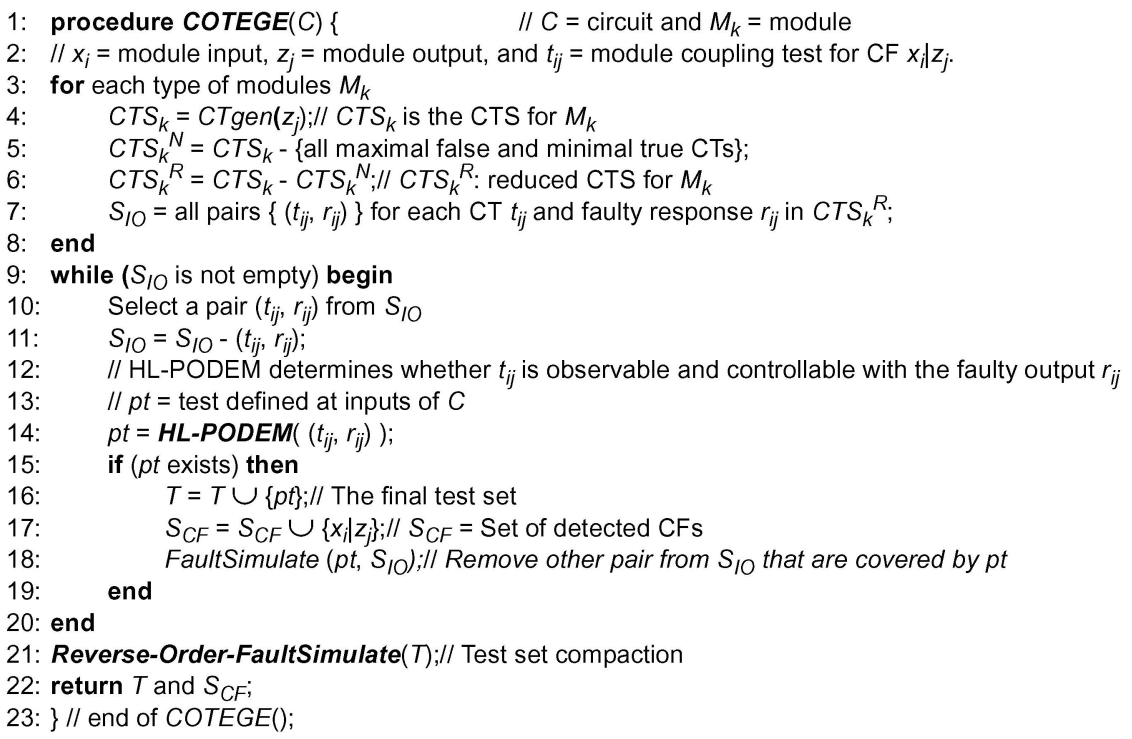

Fig. 14. Pseudocode for the COTEGE program which computes a high-level test set for a modular circuit based on the coupling fault model.

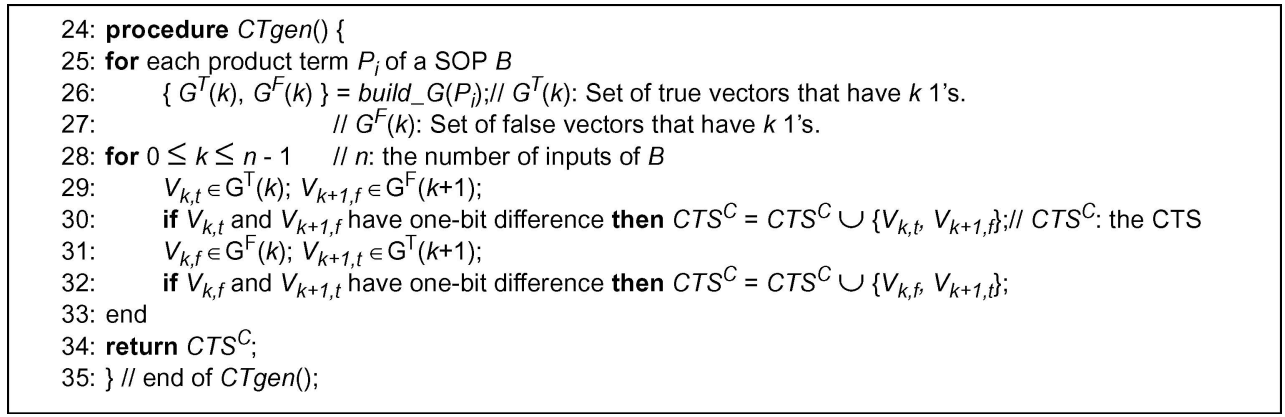

Fig. 15. Pseudocode of $C T_{\text {gen }()}$ which generates all CTs for a given logic function.

is not necessary to apply $e_{h}$ if all tests in $T_{C}$ are applied to a BIP network for stuck-at fault detection. Similarly, if $t_{i j}$ is a false vector, $T_{C}$ is the largest set $e_{k}$ of incomparable expanded false CTs that contains a CT $e_{k} \geq e_{h}$ for any false expanded CT $e_{h}<e_{i j}$. For example, consider the 3-input function $z$ whose expanded truth table is shown in Table 3. All eight input vectors are CTs and the reduced CTS for $z$ is composed of the four shaded tests. If (expanded) test 0101 is either uncontrollable or unobservable, then $T_{C}=\{0111\}$ replaces 0101 .

Computing tests via the Boolean difference is known to be hard. In Larrabee's ATPG method [15], for example, the Boolean difference is used only for a small number of hard-to-detect faults after random tests are
Table 3. Expanded truth table of the function $z=$ $a b+a^{\prime} c^{\prime}$.

\begin{tabular}{lccc}
\hline $\begin{array}{l}\text { Input vector } \\
a b c\end{array}$ & $\begin{array}{c}\text { Expanded vector } \\
a a^{\prime} b c^{\prime}\end{array}$ & $z$ \\
\hline 000 & 0101 & 1 & Min. true CT \\
001 & 0100 & 0 & \\
010 & 0111 & 1 & \\
011 & 0110 & 0 & Max. false CT \\
100 & 1001 & 0 & Max. false CT \\
101 & 1000 & 0 & \\
110 & 1011 & 1 & \\
111 & 1010 & 1 & Min. true CT \\
\hline
\end{tabular}


generated to detect easy-to-detect faults. The representation and manipulation of Boolean functions can be implemented in several ways: sum-of-products, conjunctive normal form, or binary decision diagrams (BDDs). As we show in [25], the complexity of computing the Boolean difference can be greatly reduced by using BDDs. However, in the current version of COTEGE, the CT generation procedure $C T$ gen() exhaustively searches for CTs by using the properties of adjacent CTs. In other words, all vector pairs separated by Hamming distance 1 are examined if they have different outputs. If so, the two vectors are added to the CTS. The size of a function that can be efficiently handled by COTEGE is also restricted by test set size. In our experiments, we limit the number of module inputs to at most 20 . Note that some functions still have too many tests with 20 inputs. For example, the XOR function with 20 inputs has an exhaustive set of $2^{20}$ tests, so we limit XOR modules to 6 inputs.

\section{Experimental Results}

We applied COTEGE to some representative and fairly big combinational circuits on a $400 \mathrm{MHz}$ SUN Ultra5 workstation. The test circuits for the experiments include two ISCAS-85 benchmarks (c880 and c7552), three datapath circuits (a 16-bit carry lookahead adder CLA, a 16-bit ALU, and a 16-bit magnitude comparator), and two fully binate circuits (the 20-input identity function $l_{20}$ and a 12-bit address comparator). The ISCAS-85 and datapath circuits have the module struc- ture used in [14], and are composed of modules containing as many as 17 inputs and 8 outputs. The two binate circuits are viewed as single modules. Fig. 13 shows the modular design we used for c880.

Table 4 compares the size of the reduced CTS generated by COTEGE to that of the UTS generated by RIBTEC for each example circuit. As can be seen, the size differences between the CTSs and UTSs are negligible for the CLA, ALU, magnitude comparator, c880, and $\mathrm{c7552}$. These circuits have highly unate modules, so their UTSs are also quite small. As expected, for the two fully binate functions the CTS is much smaller than the UTS, which is, of course, exhaustive. We also generated test sets for SSL faults using a conventional (non-hierarchical) ATPG tool ATALANTA [16] and two different gate-level implementations of each circuit; these implementations were synthesized either for low area or high speed by the Synopsys Design Compiler. As Table 4 shows, the ratio of the size of the CTS to that of the SSL test set ranges from 1 to 8. Interestingly, the CTS and SSL test sets for $l_{20}$ are identical for both implementations.

Conventional SSL fault simulation was performed on the synthesized implementations of each circuit with the above test sets. Note that we do not restrict the synthesis techniques to test-set preserving ones. Table 5 summarizes the results. For example, the coupling and universal test sets achieve $100 \%$ fault coverage for the 16-bit CLA circuit optimized for low area (listed under Area) and high speed (listed under Speed). The test set generated by ATALANTA for the low-area design achieves $100 \%$ fault coverage, but it misses 39 faults in the high-speed design, which results in $93 \%$ fault

Table 4. Size of the test sets generated by COTEGE, RIBTEC and ATALANTA (CPU time is in seconds.).

\begin{tabular}{|c|c|c|c|c|c|c|c|c|c|c|c|}
\hline \multirow[b]{3}{*}{ Circuits } & & & & & \multirow{3}{*}{$\begin{array}{c}\text { Test set } \\
\text { size } \\
\text { ratio } \mathrm{C} / \mathrm{R}\end{array}$} & \multicolumn{6}{|c|}{ ATALANTA (A) } \\
\hline & \multicolumn{2}{|c|}{ COTEGE (C) } & \multicolumn{2}{|c|}{ RIBTEC (R) } & & \multicolumn{3}{|c|}{ Low-area realization } & \multicolumn{3}{|c|}{ High-speed realization } \\
\hline & $\begin{array}{l}\mathrm{CPU} \\
\text { time }\end{array}$ & $\begin{array}{l}\text { Number } \\
\text { of tests }\end{array}$ & $\begin{array}{l}\text { CPU } \\
\text { time }\end{array}$ & $\begin{array}{l}\text { Number } \\
\text { of tests }\end{array}$ & & $\begin{array}{l}\text { CPU } \\
\text { time }\end{array}$ & $\begin{array}{l}\text { Number } \\
\text { of tests }\end{array}$ & $\begin{array}{c}\text { Ratio } \\
\text { C/A }\end{array}$ & $\begin{array}{l}\text { CPU } \\
\text { time }\end{array}$ & $\begin{array}{l}\text { Number } \\
\text { of tests }\end{array}$ & $\begin{array}{c}\text { Ratio } \\
\text { C/A }\end{array}$ \\
\hline 16-bit CLA & 282 & 48 & 1,254 & 48 & 1 & 0.05 & 24 & 2.0 & 0.13 & 30 & 1.6 \\
\hline 16-bit ALU & 2.28 & 87 & 4.32 & 88 & 0.99 & 0.05 & 31 & 2.8 & 0.83 & 35 & 2.5 \\
\hline 16-bit comparator & 16.3 & 110 & 12.0 & 114 & 0.96 & 0.38 & 52 & 2.1 & 0.32 & 69 & 1.6 \\
\hline c880 & 597 & 208 & 410 & 226 & 0.92 & 0.15 & 46 & 4.5 & 0.17 & 64 & 3.3 \\
\hline c7552 & 1,162 & 209 & 1,159 & 213 & 0.98 & 0.35 & 52 & 4.0 & 0.92 & 65 & 3.2 \\
\hline $\begin{array}{l}\text { 20-input identity } \\
\text { function }\end{array}$ & 27.7 & 42 & - & $2^{20}$ & 0.0004 & 0.03 & 42 & 1.0 & 0.03 & 42 & 1.0 \\
\hline $\begin{array}{l}\text { 12-bit address } \\
\text { comparator ( } 16 \text { inputs) }\end{array}$ & 5.40 & 390 & - & $2^{16}$ & 0.006 & 0.07 & 48 & 8.1 & 0.08 & 53 & 7.4 \\
\hline
\end{tabular}




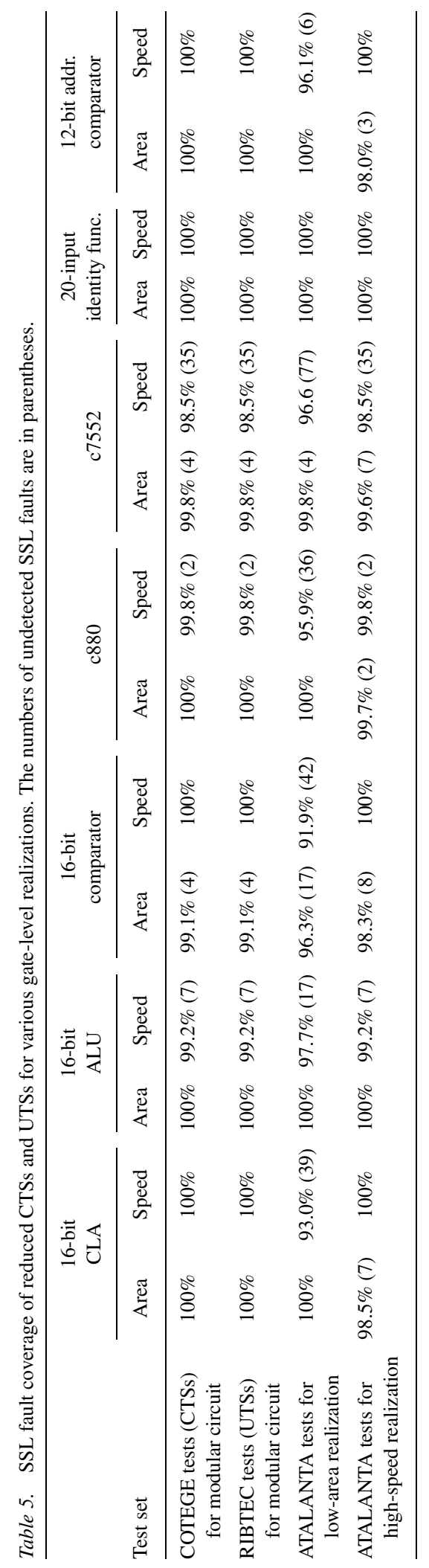




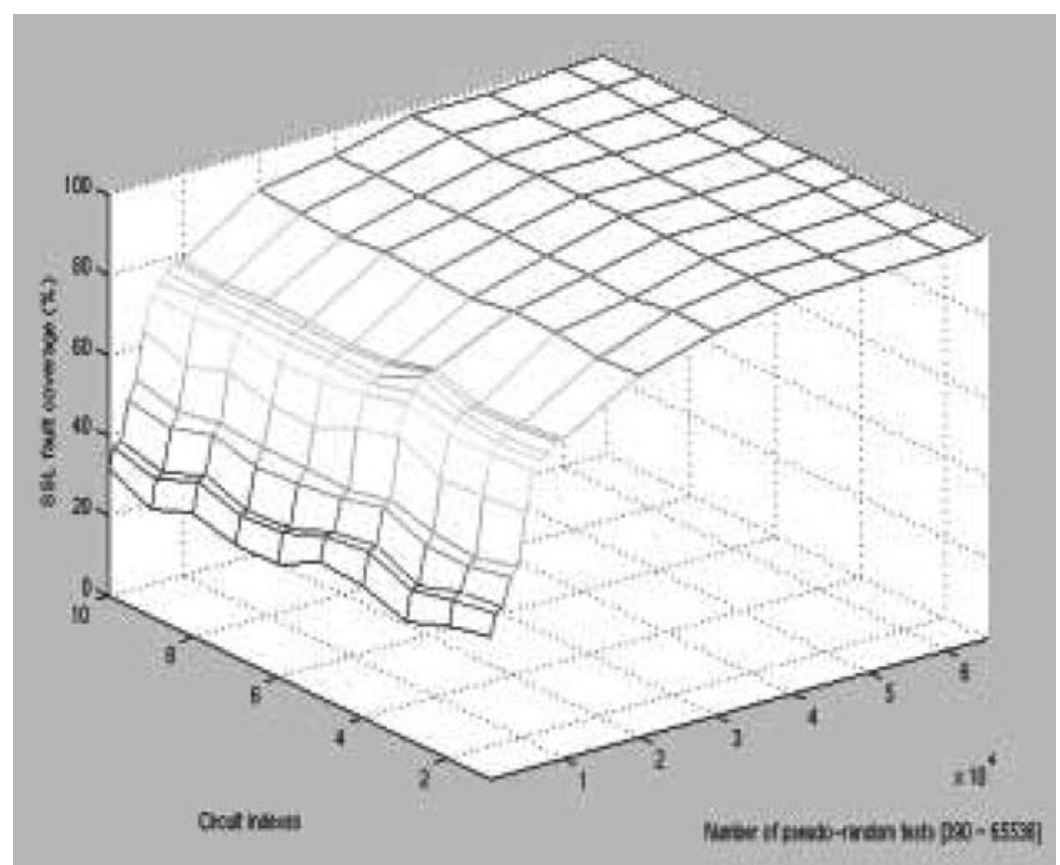

Fig. 16. SSL fault coverage of pseudorandom test sets for 10 different realizations of the address comparator.

coverage. As can be seen from Table 5, both the coupling and universal test sets detect all SSL faults in all gate-level implementations, while the realizationdependent SSL test sets for one design miss some faults in the other design. Note that the CTSs for the two binate functions detect all SSL faults in all gate-level implementations with fewer tests than the UTSs. Also, note that the high-level test sets (coupling and universal test sets) for the 16-bit magnitude comparator detect some hard-to-detect faults in the low-area design which the conventional ATPG tool (ATALANTA) aborts. In general, the SSL fault coverage of the high-level test sets is better than that of the realization-dependent test sets.

Pseudorandom testing can also provide a high degree of realization independence at the cost of a very large number of tests. Fig. 16 shows the SSL fault coverage of (pseudo) random test sets for 10 different implementations of the 12-bit address comparator. The fault coverage figures of the random test sets for all implementations are almost the same, as expected. The SSL fault coverage of 390 random vectors is 20 to $30 \%$ in all cases. To achieve coverage above $90 \%$, more than $20 \mathrm{~K}$ random test vectors are required; to achieve $100 \%$ fault coverage, more than $61 \mathrm{~K}$ random vectors were re- quired. Thus we need a near-exhaustive random test set to achieve $100 \%$ SSL fault coverage for any realization of the address comparator, while $390 \mathrm{CT}$ vectors provide $100 \%$ SSL fault coverage for all the realizations considered.

The coupling delay test sets for two fully binate functions ( $I_{20}$ and the 12-bit address comparator) and four ISCAS- 89 benchmark circuits were generated and the results are shown in Table 6. Each circuit is regarded as a single module in this experiment. For the two binate functions, the low-area implementations used in previous experiments are used to compute the number of paths and path delay faults. The number of path delay faults is the maximum number of path delay tests. The number of coupling delay tests was found to be from one to 65 times larger than that of the path delay tests. We propose a hierarchical coupling delay test generation method and a fault model in [25] to address this test-set size problem. Nevertheless, the size of the coupling delay test sets is much less than that of the corresponding gross delay test set, that is, the set of all SIC tests. (Recall that the number of SIC tests for an $n$-input function is $n \cdot 2^{n}$.) The coupling delay tests also have the advantage of detecting all robust path delay faults in any realization. 
Table 6. Coupling delay test generation results for two fully binate functions and four ISCAS-89 benchmark circuits.

\begin{tabular}{|c|c|c|c|c|c|c|c|}
\hline \multirow[b]{2}{*}{ Circuit } & \multirow[b]{2}{*}{ Description } & \multirow{2}{*}{$\begin{array}{l}\text { No. of } \\
\text { inputs }\end{array}$} & \multirow{2}{*}{$\begin{array}{l}\text { No. of path } \\
\text { delay faults }\end{array}$} & \multicolumn{3}{|c|}{ Coupling delay testing } & \multirow{2}{*}{$\begin{array}{l}\text { No. of gross } \\
\text { delay tests }\end{array}$} \\
\hline & & & & No. of faults & No. of tests & CPU time & \\
\hline$I_{20}$ & 20-input identity & 20 & 80 & 80 & 80 & $10.89 \mathrm{sec}$ & $20.9 \times 10^{6}$ \\
\hline addrcomp & 12-bit address comparator & 16 & 142 & 790 & 790 & $1.09 \mathrm{sec}$ & $1.05 \times 10^{6}$ \\
\hline s298 & Traffic light controller & 17 & 462 & 1856 & 672 & $0.3 \mathrm{sec}$ & $2.23 \times 10^{6}$ \\
\hline $\mathrm{s} 400$ & & 24 & 896 & 83,202 & 51,870 & $54.86 \mathrm{sec}$ & $403 \times 10^{6}$ \\
\hline s386 & Controller & 13 & 414 & 6,000 & 3,084 & $1.15 \mathrm{sec}$ & $0.106 \times 10^{6}$ \\
\hline s1494 & & 14 & 1,952 & 186,398 & 55,830 & $316.54 \mathrm{sec}$ & $0.229 \times 10^{6}$ \\
\hline
\end{tabular}

\section{Conclusions}

A high-level fault model called the coupling fault (CF) model has been proposed to address some testing problems that cannot be efficiently handled at the gate level. The basic properties of CFs and their tests have been explored, one of which is the ability to cover both functional and delay faults in a uniform and realization-independent manner. A test generation program COTEGE has been developed for CF detection. Our experiments with COTEGE show that (reduced) coupling test sets efficiently cover standard, low-level faults in a variety of realizations using fewer tests than other high-level test sets. The corresponding coupling delay tests (CDTs) detect all robust path delay faults in any realization of a function. CDT sets tend to be larger than typical path delay test sets, but their size can be reduced by employing hierarchical methods [25].

\section{References}

1. S.B. Akers, "Universal Test Sets for Logic Networks," IEEE Trans. on Computers, vol. C-22, no. 9, pp. 835-839, 1973.

2. H. Al-Asaad, J.P. Hayes, and B.T. Murray, "Scalable Test Generators for High-Speed Datapath Circuits," Journal of Electronic Testing, vol. 12, pp. 111-125, 1998.

3. M.J. Batek and J.P. Hayes, "Test-Set Preserving Logic Transformations," in Proc. Design Automation Conf., pp. 454-458, 1992.

4. R. Betancourt, "Derivation of Minimum Test Sets for Unate Logic Circuits," IEEE Trans. on Computers, vol. C-20, no. 11, pp. 1264-1269, 1971.

5. K.-T. Cheng, A. Krstic, and H-C Chen, "Generation of High Quality Tests for Robustly Untestable Path Delay Faults," IEEE Trans. on Computers, vol. 45, no. 12, pp. 1379-1392, 1996.

6. K. De, "Test Methodology for Embedded Cores which Protects Intellectual Property," in Proc. VLSI Test Symposium, 1997, pp. 2-9
7. B.I. Dervisoglu, "A Unified DFT Architecture for Use with IEEE 1149.1 and VSIA/IEEE P1500 Compliant Test Access Controllers," in Proc. Design Automation Conference, pp. 53-58, 2001.

8. S. Devadas and K. Keutzer, "Synthesis of Robust Delay-FaultTestable Circuits: Theory," IEEE Trans. on CAD, vol. 11, no. 1, pp. 87-101, 1992.

9. M. Gharaybeh, M.L. Bushnell, and V.D. Agrawal, "Classification and Test Generation for Path-Delay Faults Using Single Stuck-at Fault Tests," Journal of Electronic Testing, vol. 11, pp. 55-67, 1997.

10. P. Goel, "An Implicit Enumeration Algorithm to Generate Tests for Combinational Logic Circuits," IEEE Trans. on Computers, vol. C-30, no. 3, pp. 215-222, 1981.

11. J.P. Hayes, "On Realizations of Boolean Functions Requiring a Minimal or Near-Minimal Number of Tests," IEEE Trans. on Computers, vol. C-20, no. 12, pp. 1506-1513, 1971.

12. W. Ke and P.R. Menon, "Synthesis of Delay-Verifiable Combinational Circuits," IEEE Trans. on Computers, vol. 44, no. 2, 1995.

13. H. Kim and J.P. Hayes, "Delay Fault Testing of Designs with Embedded IP Cores," in Proc. VLSI Test Symposium, 1999, pp. 160167.

14. H. Kim and J.P. Hayes, "Realization-Independent ATPG for Designs with Unimplemented Blocks," IEEE Trans. on CAD, vol. 20, no. 2, pp. 290-306, 2001.

15. T. Larrabee, "Efficient Generation of Test Patterns Using Boolean Difference," in Proc. International Test Conf., pp. 795801, 1989.

16. H.K. Lee and D.S. Ha, "On the Generation of Test Patterns for Combinational Circuits," Tech. Report 12-93, EE Dept., Virginia Polytechnic Institute and State University, 1993.

17. C.J. Lin and S.M. Reddy, "On Delay Fault Testing in Logic Circuits," IEEE Trans. on CAD, vol. CAD-6, no. 5, pp. 694 703, 1987.

18. I. Pomeranz and S.M. Reddy, "On Testing Delay Faults in Macro-Based Combinational Circuits," in Proc. International Conf. on CAD, pp. 332-339, 1994.

19. I. Pomeranz and S.M. Reddy, "Functional Test Generation for Delay Faults in Combinational Circuits," in Proc. International Conf. on CAD, pp. 687-694, 1995.

20. M. Psarakis, D. Gizopoulos, and A. Paschalis, "Test Generation and Fault Simulation for Cell Fault Model Using Stuck-at Fault Model Based Test Tools," Journal of Electronic Testing, vol. 13, pp. 315-319, 1998. 
21. M. Psarakis et al., "Sequential Fault Modeling and Test Pattern Generation for CMOS Iterative Logic Arrays," IEEE Trans. on Computers, vol. 49, no. 10, pp. 1083-1099, 2000.

22. J. Rajski and J. Vasudevamurthy, "The Testability-Preserving Concurrent Decomposition and Factorization of Boolean Expressions," IEEE Trans. on CAD, vol. 11, no. 6, pp. 778-793, 1992.

23. G.L. Smith, "Model for Delay Faults Based Upon Paths," in Proc. International Test Conf., pp. 342-349, 1985.

24. U. Sparmann, H. Muller, and S.M. Reddy, "Universal Delay Test Sets for Path Delay Faults," IEEE Trans. on VLSI Systems, vol. 7, no. 2, pp. 156-166, 1999.

25. J. Yi, "High-Level Function and Delay Testing for Digital Circuits," Ph.D Thesis, University of Michigan, 2002.

Joonhwan Yi received the B.S degree in electronics engineering from Yonsei University, Seoul, Korea, in 1991, and the M.S. and $\mathrm{Ph} . \mathrm{D}$ degrees in electrical engineering and computer science from the University of Michigan, Ann Arbor, in 1998 and 2002, respectively.

From 1991 to 1995, he was with Samsung Electronics, Semiconductor Business, Korea, where he was involved in developing application specific integrated circuit cell libraries. In 2000, he was a summer intern with Cisco, Santa Clara, CA, where he worked for path delay fault testing. Since 2003, he has been with Samsung Electronics, Telecommunication Network, Suwon, Korea, where he is working on system-on-a-chip design. His current research interests include C-level system modeling for fast hardware and software cosimulation, system-level power analysis and optimization, behavioral synthesis, and high-level testing.
John P. Hayes received the B.E. degree from the National University of Ireland, Dublin, and the M.S. and Ph.D. degrees from the University of Illinois, Urbana-Champaign, all in electrical engineering. While at the University of Illinois, he participated in the design of the ILLIAC III computer. In 1970 he joined the Operations Research Group at the Shell Benelux Computing Center in The Hague, where he worked on mathematical programming and software development. From 1972 to 1982 he was a faculty member of the Departments of Electrical Engineering-Systems and Computer Science of the University of Southern California, Los Angeles. Since 1982 he has been with the Electrical Engineering and Computer Science Department of the University of Michigan, Ann Arbor, where he holds the Claude E. Shannon Chair in Engineering Science.

Professor Hayes was the Founding Director of the University of Michigan's Advanced Computer Architecture Laboratory (ACAL). He has authored over 225 technical papers, several patents, and five books, including Introduction to Digital Logic Design (AddisonWesley, 1993), and Computer Architecture and Organization, (3rd edition, McGraw-Hill, 1998). He has served as editor of various technical journals, including the Communications of the ACM, the IEEE Transactions on Parallel and Distributed Systems and the Journal of Electronic Testing. Professor Hayes is a fellow of both IEEE and ACM, and a member of Sigma Xi. He received the University of Michigan's Distinguished Faculty Achievement Award in 1999 and the Humboldt Foundation's Research Award in 2004. His current teaching and research interests are in the areas of computer-aided design, verification, and testing; VLSI circuits; faulttolerant embedded systems; ad-hoc computer networks; and quantum computing. 